\title{
Le Professeur Adolphe Dupont
}

Le Professeur Adolphe Dupont s'est éteint à Namur le 5 juin 1982, dans Thôtel familial qu'il affectionnait et où il exerça la dermatologie pendant plus de 50 ans.

Né dans cette jolie ville mosane le 24 mai 1898, il y accomplit ses humanités au College NotreDame de la Paix. II s'inscrivit en-suite à $\Gamma$ Université catholique de Louvain et fut promu docteur en médecine en 1925. II suivit pendant quelques mois les travaux de la Clinique dermatologique du Professeur Aimé Morelle à Louvain, puis alia passer un an à Strasbourg auprès du Professeur L.-M. Pautrier. Ensuite ce fut Paris et $\Gamma$ Hôpital Saint-Louis, où il devint Гélève d'Achille Civatte. Ces deux stages devaient le marquer de façon indélébile pour le restant de sa vie.

Enthousiaste, passionné, intransigeant, il avait puisé chez ses maîtres Pautrier et Ci-vatte la rigueur clinique et la connaissance approfondie de $\Gamma$ anatomie pathologique. In-troduit par le Docteur Paul Francois, direc-teur de THôpital dermatologique Notte-bohm, il devint membre de la Société beige de Dermatologie en 1930. Francois et lui 1 Dans la redaction de cet hommage à la mémoire du Professeur Dupont, nous avons fait appel tant à nos souvenirs personnels qu'aux témoignages de MM. Bour-lond et Lachapelle, ses anciens disciples, à qui nous ex-primons notre gratitude pour leur aimable collaboration.

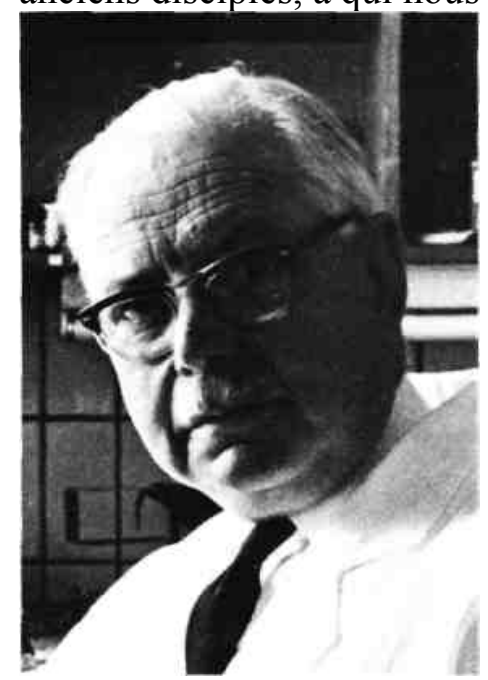

s'étaient connus à Anvers où Dupont assu-rait la consultation de dermatologie de ГHôpital SaintVincent, consultation à la-quelle il resta fidèle jusqu'à la guerre de 1940. Médecin de reserve, il repritdu service actifàlafmde 1944etàpartirde 1945, il di-rigea le Centre dermato-vénéréologique de I'Hôpital militaire de Namur. II n'acceda que tardivement au professorat, car ce n'est qu'en 1955 qu'il put prendre, à $\Gamma$ Université catholique de Louvain, la succession du Professeur N. Yernaux, atteint par la limite d'âge 2 ans auparavant.

Dès son accession à la chaire, il imprima à son service un rythme de travail intensif et lui acquit rapidement une brillante noto-riété. II n'était d'ailleurs pas un inconnu

Nécrologie 
dans le monde dermatologique et ses travaux antérieurs faisaient autorité. Dupont a beau-coup publié, et aucun de ses articles n'est indifferent. Son intérêt allait avant tout à This-topathologie, mais pas seulement cutanée, car il avait de solides notions d'anatomie pa-thologique générale. II fut le premier à don-ner, à la Société beige de Dermatologie, droit de cite à Гétude histologique systématique des affections cutanées. II s'est intéressé par-ticulièrement aux tumeurs et aux derma-toses bulleuses. II écrivit des articles sur Гépithélioma calcifié de Malherbe, les tumeurs sudoripares, le kérato-acanthome, Гépithélioma spinocellulaire, Гacantholyse dans les pemphigus et la maladie de Hailey-Hailey. On lui doit la premiere observation de ce qu'on appelle à present la «papulose lymphomatoïde», ainsi que Гobservation princeps, en collaboration avec J.M. Lacha-pelle, de la thésaurismose cutanée par la polyvinylpyrrolidone (maladie de Dupont et Lachapelle). Sa these d'agrégation, en 1951, sur «L'angioréticulomatose cutanée (Sar-come multiple idiopathique pigmentaire de Kaposi)» couronnait une série d'articles déjà consacrés à cette affection, et ses travaux sur Thistologie et $\Gamma$ histopathologie du système nerveux de la peau (poursuivis actuellement par son successeur, le Professeur Bourland) lui permirent de réunir à Louvain, en une seance spéciale, les meilleurs spécialistes eu-ropéens de la question.

Dupont écrivait une langue scientifique claire et precise; s'attachant toujours à $\Gamma$ es-sentiel et ne se perdant jamais dans des details. Membre titulaire de la Société fran-çaise de dermatologie, il était également membre d'honneur de plusieurs sociétés eu-ropéennes de Dermatologie et Docteur honoris causa de $\Gamma$ Université de Besançon. II fut president de la Société beige de Dermatologie et de la Société beige antivénérienne.

Jusqu'à la fin de sa vie son intérêt pour les reunions scientifiques était demeuré ex-trêmement vivace. Fidèle en amitié, il était resté profondément attache à la clinique dermatologique de Strasbourg, où il se ren-dait fréquemment, de même qu'aux seances de la Société française de Dermatologie.

Dupont avait un temperament d'artiste pictural. Ses croquis, ses rares eaux-fortes sont d'excellente facture et il s'est adonné à la peinture avec talent. Très entier dans ses convictions, qu'il énonçait avec force et passion, il avait des idées très arrêtées sur Tart contemporain, qu'il dédaignait en bloc, ré-servant ses admirations pour certains pein-tres d'autrefois. II était très attache à la conservation du patrimoine artistique de notre pays et fut pendant de longues années le President écouté de la Société archéologique de Namur.

C. C'est une belle figure de la dermatologie beige qui a disparu.

J. Piérard Marg. Ledoux 